\title{
Stability of Methods for Matrix Inversion
}

\author{
Jeremy J. Du Croz * Nicholas J. Higham ${ }^{\dagger}$ \\ May 7, 1991 In IMA J. Numer. Anal., 12 (January 1992).
}

\begin{abstract}
Inversion of a triangular matrix can be accomplished in several ways. The standard methods are characterised by the loop ordering, whether matrix-vector multiplication, solution of a triangular system, or a rank-1 update is done inside the outer loop, and whether the method is blocked or unblocked. The numerical stability properties of these methods are investigated. It is shown that unblocked methods satisfy pleasing bounds on the left or right residual. However, for one of the block methods it is necessary to convert a matrix multiplication into the solution of a multiple right-hand side triangular system in order to have an acceptable residual bound. The inversion of a full matrix given a factorization $P A=L U$ is also considered, including the special cases of symmetric indefinite and symmetric positive definite matrices. Three popular methods are shown to possess satisfactory residual bounds, subject to a certain requirement on the implementation, and an attractive new method is described. This work was motivated by the question of what inversion methods should be used in LAPACK.
\end{abstract}

Key words: matrix inversion, triangular matrix, error analysis, block algorithm, LAPACK.

AMS(MOS) subject classifications. primary 65F05, 65G05.

\footnotetext{
*Numerical Algorithms Group Ltd., Wilkinson House, Jordan Hill Road, Oxford, OX2 8DR. (nagjdc@vax.oxford.ac.uk).

${ }^{\dagger}$ Department of Mathematics, University of Manchester, Manchester, M13 9PL, UK. (mbbgsnh@cms.mcc.ac.uk).
} 


\section{Introduction}

As Forsythe, Malcolm and Moler [8, p .31] point out, "In the vast majority of practical computational problems, it is unnecessary and inadvisable to actually compute $A^{-1}$." Nevertheless, there are some applications that genuinely require computation of a matrix inverse-see [1, sec. 7.5], [14, p. 342ff] and [4,10] for example. LAPACK [3], like LINPACK before it, will include routines for matrix inversion. LAPACK will support inversion of triangular matrices and of general, symmetric indefinite, and symmetric positive definite matrices via an $L U$ (or related) factorization. Each of these matrix inversions can be done in several ways. For example, in triangular matrix inversion different loop orderings are possible and either triangular matrix-vector multiplication, solution of a triangular system, or a rank-1 update of a rectangular matrix can be employed inside the outer loop. As a further example, given a factorization $P A=L U$, two ways to evaluate $A^{-1}$ are as $A^{-1}=U^{-1} \times L^{-1} \times P$, and as the solution to $U A^{-1}=L^{-1} \times P$. These methods generally achieve different levels of efficiency on high-performance computers, and they propagate rounding errors in different ways. The performance issues are fairly well understood. The purpose of this work is to investigate the numerical stability properties of the methods, with a view to guiding the choice of inversion method in LAPACK.

Existing error analysis, such as that in $[18,20]$ and [11], is applicable to two of the methods considered here (Method 1 and Method A). We believe our analysis for the other methods to be new. A secondary aim of this work is to use matrix inversion as a vehicle for illustrating some important principles in error analysis. Our strategy is to determine what sorts of error bounds we can expect to prove, do the error analysis in a concise and modular fashion, and then gain further insight from numerical tests.

The quality of an approximation $Y \approx A^{-1}$ can be assessed by looking at the right and left residuals $A Y-I$ and $Y A-I$, and the forward error, $Y-A^{-1}$. Suppose we perturb $A \rightarrow A+\Delta A$ with $|\Delta A| \leq \epsilon|A|$, where the absolute value and the inequality hold componentwise; thus we are making relative perturbations of size at most $\epsilon$ to the elements of $A$. If $Y=(A+\Delta A)^{-1}$ then $(A+\Delta A) Y=Y(A+\Delta A)=I$, so that

$$
\begin{aligned}
& |A Y-I|=|\Delta A Y| \leq \epsilon|A||Y|, \\
& |Y A-I|=|Y \Delta A| \leq \epsilon|Y||A|,
\end{aligned}
$$


and, since $(A+\Delta A)^{-1}=A^{-1}-A^{-1} \Delta A A^{-1}+O\left(\epsilon^{2}\right)$,

$$
\left|A^{-1}-Y\right| \leq \epsilon\left|A^{-1}\right|\left|A \| A^{-1}\right|+O\left(\epsilon^{2}\right)
$$

(Note that (1.3) can also be derived from (1.1) or (1.2).) The bounds (1.1)-(1.3) represent "ideal" bounds for a computed approximation $Y$ to $A^{-1}$ if we regard $\epsilon$ as a small multiple of the unit roundoff $u$. We will show that, for triangular matrix inversion, appropriate methods do indeed achieve (1.1) or (1.2) (but not both) and (1.3).

We stress that neither (1.1), (1.2) nor (1.3) implies that $Y+\Delta Y=(A+\Delta A)^{-1}$ with $\|\Delta A\|_{\infty} \leq \epsilon\|A\|_{\infty}$ and $\|\Delta Y\|_{\infty} \leq \epsilon\|Y\|_{\infty}$, that is, $Y$ need not be close to the inverse of a matrix near to $A$, even in the norm sense. Indeed, such a result would imply that both the left and right residuals are bounded in norm by $\left(2 \epsilon+\epsilon^{2}\right)\|A\|_{\infty}\|Y\|_{\infty}$, and this is not the case for any of the methods we will consider. See [15, pp. 375-377] and [18, sec. 26] for more on this aspect of the stability of matrix inversion.

We will use the following model of floating point arithmetic:

$$
\begin{aligned}
& f l(x \pm y)=x(1+\alpha) \pm y(1+\beta), \quad|\alpha|,|\beta| \leq u \\
& f l(x \text { op } y)=(x \text { op } y)(1+\delta), \quad|\delta| \leq u, \quad \text { op }=*, / .
\end{aligned}
$$

We quote the standard result that if $L \in \mathbb{R}^{n \times n}$ is lower triangular then forward substitution applied to $L x=b$ produces a computed solution $\widehat{x}$ that satisfies (see, for example, [17, pp. 150,408])

$$
(L+\Delta L) \hat{x}=b, \quad|\Delta L| \leq c_{n} u|L| .
$$

Here, and below, we use $c_{n}$ to denote a constant of order $n$. We are not concerned with the precise values of the constants in the analysis. (See [19, pp. 102,108] for some comments on the interpretation of the constants.)

To simplify the presentation we introduce a special notation. Let $A_{i} \in \mathbb{R}^{m_{i} \times n_{i}}$, $i=1: k$, be matrices such that the product $A_{1} A_{2} \cdots A_{k}$ is defined and let

$$
p=\sum_{i=1}^{k-1} n_{i} .
$$

Then $\Delta\left(A_{1}, A_{2}, \ldots, A_{k}\right) \in \mathbb{R}^{m_{1} \times n_{k}}$ denotes a matrix bounded according to

$$
\left|\Delta\left(A_{1}, A_{2}, \ldots, A_{k}\right)\right| \leq c_{p} u\left|A_{1}\right|\left|A_{2}\right| \cdots\left|A_{k}\right|+O\left(u^{2}\right)
$$


This notation is chosen so that if $\hat{C}=f l\left(A_{1} A_{2} \cdots A_{k}\right)$, with the product evaluated in any order, then

$$
\widehat{C}=A_{1} A_{2} \ldots A_{k}+\Delta\left(A_{1}, A_{2}, \ldots, A_{k}\right),
$$

as is easily verified. Note also that the matrix $\Delta L$ in (1.4) can be expressed as $\Delta(L)$, if we define $p=n_{1}$ when $k=1$.

We consider the inversion of triangular matrices in section 2. The inversion of full matrices is treated in section 3 , and conclusions are given in section 4 .

\section{Inverting a Triangular Matrix}

We consider the inversion of a lower triangular matrix $L \in \mathbb{R}^{n \times n}$, treating unblocked and blocked methods separately.

\subsection{Unblocked Methods}

We focus our attention on two " $j$ " methods that compute $L^{-1}$ a column at a time. Analogous " $i$ " and " $k$ " methods exist, which compute $L^{-1}$ row-wise or use outer products, respectively, and we comment on them at the end of the section. (The names " $i$ ", " $j$ " and " $k$ " refer to the outermost loop index, according to the convention introduced by [7], and used in [9], to describe the different possible orderings of the loops.)

The first method computes each column of $X=L^{-1}$ independently, using conventional forward substitution. We write it as follows, to facilitate comparison with the second method. We use MATLAB-style indexing notation, as in [9].

\section{Method 1.}

for $j=1: n$

$$
\begin{aligned}
& x_{j j}=l_{j j}^{-1} \\
& X(j+1: n, j)=-x_{j j} L(j+1: n, j)
\end{aligned}
$$

Solve $L(j+1: n, j+1: n) X(j+1: n, j)=X(j+1: n, j)$ by forward substitution end 
In BLAS terminology, this method is dominated by $n$ calls to a level 2 BLAS routine xTRSV (TRiangular SolVe).

The second method computes the columns in the reverse order. On the $j$ th step it multiplies by the previously computed inverse $L(j+1: n, j+1: n)^{-1}$ instead of solving a system with coefficient matrix $L(j+1: n, j+1: n)$.

\section{Method 2.}

for $j=n:-1: 1$

$$
\begin{aligned}
& x_{j j}=l_{j j}^{-1} \\
& X(j+1: n, j)=X(j+1: n, j+1: n) L(j+1: n, j) \\
& X(j+1: n, j)=-x_{j j} X(j+1: n, j)
\end{aligned}
$$

end

Method 2 uses $n$ calls to the level 2 BLAS routine xTRMV (TRiangular Matrix times Vector). On most high-performance machines XTRMV can be implemented to run faster than xTRSV, so Method 2 is generally preferable to Method 1 from the point of view of efficiency (see the performance figures at the end of section 2.2). We now compare the stability of the two methods.

The result (1.4) shows that the $j$ th column of the computed $\widehat{X}$ from Method 1 satisfies

$$
\left(L+\Delta L_{j}\right) \widehat{x}_{j}=e_{j}, \quad\left|\Delta L_{j}\right| \leq c_{n} u|L| .
$$

It follows that we have the componentwise residual bound

$$
|L \widehat{X}-I| \leq c_{n} u|L||\widehat{X}|
$$

and the componentwise forward error bound

$$
\left|\widehat{X}-L^{-1}\right| \leq c_{n} u\left|L^{-1}\right||L||\widehat{X}|
$$

Since $\widehat{X}=L^{-1}+O(u),(2.2)$ can be written as

$$
\left|\widehat{X}-L^{-1}\right| \leq c_{n} u\left|L^{-1}\right||L|\left|L^{-1}\right|+O\left(u^{2}\right)
$$

which is invariant under row and column scaling of $L$. If we take norms we obtain normwise relative error bounds that are either row or column scaling independent: 
from (2.3) we have

$$
\frac{\left\|\widehat{X}-L^{-1}\right\|_{\infty}}{\left\|L^{-1}\right\|_{\infty}} \leq c_{n} u \operatorname{cond}\left(L^{-1}\right)+O\left(u^{2}\right),
$$

where $\operatorname{cond}(A)=\left\|\left|A^{-1}\right||A|\right\|_{\infty}$ is the condition number of Bauer [2] and Skeel [16], and the same bound holds with cond $\left(L^{-1}\right)$ replaced by cond $(L)$.

Notice that $(2.1)$ is a bound for the right residual, $L \widehat{X}-I$. This is because Method 1 is derived by solving $L X=I$. Conversely, Method 2 can be derived by solving $X L=I$, which suggests that we should look for a bound on the left residual for this method.

Lemma 2.1 The computed inverse $\widehat{X}$ from Method D satisfies

$$
|\widehat{X} L-I| \leq c_{n} u|\widehat{X}||L|+O\left(u^{2}\right)
$$

Proof. The proof is by induction on $n$, the case $n=1$ being trivial. Assume the result is true for $n-1$ and write

$$
L=\left[\begin{array}{cc}
\alpha & 0 \\
y & M
\end{array}\right], \quad X=L^{-1}=\left[\begin{array}{cc}
\beta & 0 \\
z & N
\end{array}\right],
$$

where $\alpha, \beta \in \mathbb{R}, y, z \in \mathbb{R}^{n-1}$ and $M, N \in \mathbb{R}^{(n-1) \times(n-1)}$. Method 2 computes the first column of $X$ by solving $X L=I$ according to

$$
\beta=\alpha^{-1}, \quad z=-\beta N y \text {. }
$$

In floating point arithmetic we obtain

$$
\begin{aligned}
\hat{\beta} & =\alpha^{-1}(1+\delta), \quad|\delta| \leq u, \\
\widehat{z} & =-\widehat{\beta} \widehat{N} y+\Delta(\widehat{\beta}, \widehat{N}, y) .
\end{aligned}
$$

Thus

$$
\begin{aligned}
\hat{\beta} \alpha & =1+\delta \\
\widehat{z} \alpha+\widehat{N} y & =-\delta \widehat{N} y+\alpha \Delta(\widehat{\beta}, \widehat{N}, y)
\end{aligned}
$$

This may be written as

$$
\begin{aligned}
|\widehat{X} L-I|(1: n, 1) & \leq\left[\begin{array}{c}
u \\
u|\widehat{N}||y|+c_{n} u(1+u)|\widehat{N}||y|
\end{array}\right]+O\left(u^{2}\right) \\
& \leq c_{n}^{\prime}(|\widehat{X}||L|)(1: n, 1)+O\left(u^{2}\right) .
\end{aligned}
$$

By assumption the corresponding inequality holds for the $(2: n, 2: n)$ submatrices and so the result is proved. 
Lemma 2.1 shows that Method 2 has a left residual analogue of the right residual bound (2.1) for Method 1. From (2.5) we obtain the forward error bound

$$
\left|\widehat{X}-L^{-1}\right| \leq c_{n} u|\widehat{X}||L|\left|L^{-1}\right|+O\left(u^{2}\right),
$$

which is essentially the same as $(2.2)$, since $\widehat{X}=L^{-1}+O(u)$.

Since there is in general no reason to choose between a small right residual and a small left residual, our conclusion is that Methods 1 and 2 have equally good numerical stability properties. In fact, more is true: the two methods are "equivalent", in the sense explained in the following result.

Lemma 2.2 Let $L \in \mathbb{R}^{n \times n}$ be a lower triangular matrix and let $J \in \mathbb{R}^{n \times n}$ be the exchange matrix, that is, the matrix obtained by reversing the order of the columns of the identity matrix. Suppose that in Method 1 the triangular solves use multiplication by the reciprocals of the diagonal elements rather than division by these elements (thus the only divisions in Method 1 are those to form the reciprocals in the first place). Then Method 2 applied to $L$ is equivalent to Method 1 applied to $J L^{T} J$, in the sense that exactly the same arithmetic operations are performed, although possibly in a different order.

Proof. Instead of proving the result we will simply verify it for $n=3$. We have

$$
\bar{L}=J L^{T} J=\left[\begin{array}{lll}
l_{33} & & \\
l_{32} & l_{22} & \\
l_{31} & l_{21} & l_{11}
\end{array}\right] .
$$

Method 1 computes the first column of $\bar{L}^{-1}$ as

$$
\left[l_{33}^{-1}, \quad-l_{22}^{-1} l_{33}^{-1} l_{32}, \quad l_{11}^{-1}\left(-l_{33}^{-1} l_{31}-l_{21}\left(-l_{22}^{-1} l_{33}^{-1} l_{32}\right)\right)\right]^{T} .
$$

On its first two stages Method 2 computes $N=L(2: 3,2: 3)^{-1}$ as

$$
N=\left[\begin{array}{cc}
l_{22}^{-1} & \\
-l_{22}^{-1} l_{33}^{-1} l_{32} & l_{33}^{-1}
\end{array}\right] .
$$

Then it obtains the first column of $L^{-1}$ via

$$
L^{-1}(2: 3,1)=-l_{11}^{-1} N\left[\begin{array}{l}
l_{21} \\
l_{31}
\end{array}\right] \text {. }
$$

It is easy to see from (2.7), (2.8) and (2.9) that the same algebraic expressions are used to produce $L^{-1}(3,1)$ and $\bar{L}^{-1}(3,1)$, and $L^{-1}(2,1)$ and $\bar{L}^{-1}(3,2)=N(2,1)$. 
Lemma 2.2 implies that Method 2 satisfies the same residual bound as Method 1, modulo the $L \rightarrow J L^{T} J$ transformation, and so provides an alternative derivation of (2.5), from (2.1). Another way to express Lemma 2.2 is to say that there exist implementations of Methods 1 and 2 such that Method 2 applied to $L$ yields identical rounding errors to Method 1 applied to $J L^{T} J$. If the reciprocation assumption in Lemma 2.2 does not hold, or if we do not specify whether the column scaling should precede or follow the level 2 BLAS operation in Methods 1 and 2, then the methods will in general sustain different rounding errors but will satisfy the same residual bounds (modulo the transformation). More generally, it can be shown that all three $i, j$ and $k$ inversion variants that can be derived from the equations $L X=I$ produce identical rounding errors under suitable implementations, and all satisfy the same right residual bound; likewise, the three variants corresponding to the equation $X L=I$ all satisfy the same left residual bound. The LINPACK routine xTRDI uses a $k$ variant derived from $X L=I$; the LINPACK routines XGEDI and xPODI contain analogous code for inverting an upper triangular matrix (but [6, Chs. 1 and 3$]$ describes a different variant from the one used in the code).

\subsection{Block Methods}

Let the lower triangular matrix $L \in \mathbb{R}^{n \times n}$ be partitioned in block form as

$$
L=\left[\begin{array}{cccc}
L_{11} & & & \\
L_{21} & L_{22} & & \\
\vdots & & \ddots & \\
L_{N 1} & \ldots & \ldots & L_{N N}
\end{array}\right]
$$

where we place no restrictions on the block sizes, other than to require the diagonal blocks to be square. The most natural block generalizations of Methods 1 and 2 are as follows. Here, we use the notation $L_{p: q, r: s}$ to denote the submatrix comprising the intersection of block rows $p$ to $q$ and block columns $r$ to $s$ of $L$. 


\section{Method 1B.}

for $j=1: N$

$X_{j j}=L_{j j}^{-1}($ by Method 1$)$

$X_{j+1: N, j}=-L_{j+1: N, j} X_{j j}$

Solve $L_{j+1: N, j+1: N} X_{j+1: N, j}=X_{j+1: N, j}$ by forward substitution end

\section{Method 2B.}

for $j=N:-1: 1$

$$
\begin{aligned}
& \left.X_{j j}=L_{j j}^{-1} \text { (by Method } 2\right) \\
& X_{j+1: N, j}=X_{j+1: N, j+1: N} L_{j+1: N, j} \\
& X_{j+1: N, j}=-X_{j+1: N, j} X_{j j}
\end{aligned}
$$

end

One can argue that Method 1B carries out the same arithmetic operations as Method 1, although possibly in a different order, and that it therefore satisfies the same error bound (2.1). For completeness, we give a direct proof.

Lemma 2.3 The computed inverse $\widehat{X}$ from Method $1 B$ satisfies

$$
|L \widehat{X}-I| \leq c_{n} u|L||\widehat{X}|+O\left(u^{2}\right)
$$

Proof. Equating block columns in (2.11), we obtain the $N$ independent inequalities

$$
\left|L \widehat{X}_{1: N, j}-I_{1: N, j}\right| \leq c_{n} u|L|\left|\widehat{X}_{1: N, j}\right|+O\left(u^{2}\right), \quad j=1: N .
$$

It suffices to verify the inequality with $j=1$. Write

$$
L=\left[\begin{array}{ll}
L_{11} & \\
L_{21} & L_{22}
\end{array}\right], \quad X=\left[\begin{array}{ll}
X_{11} & \\
X_{21} & X_{22}
\end{array}\right],
$$

where $L_{11}, X_{11} \in \mathbb{R}^{r \times r}$, and $L_{11}$ is the $(1,1)$ block in the partitioning of (2.10). $X_{11}$ is computed by Method 1 and so, from (2.1),

$$
\left|L_{11} \widehat{X}_{11}-I\right| \leq c_{r} u\left|L_{11}\right|\left|\widehat{X}_{11}\right|=c_{r} u(|L||\widehat{X}|)_{11}
$$


$X_{21}$ is computed by forming $T=-L_{21} X_{11}$ and solving $L_{22} X_{21}=T$. The computed $\widehat{X}_{21}$ satisfies

$$
L_{22} \widehat{X}_{21}+\Delta\left(L_{22}, \widehat{X}_{21}\right)=-L_{21} \widehat{X}_{11}+\Delta\left(L_{21}, \widehat{X}_{11}\right)
$$

Hence

$$
\begin{aligned}
\left|L_{21} \widehat{X}_{11}+L_{22} \widehat{X}_{21}\right| & \leq c_{n} u\left(\left|L_{21}\right|\left|\widehat{X}_{11}\right|+\left|L_{22}\right|\left|\widehat{X}_{21}\right|\right)+O\left(u^{2}\right) \\
& =c_{n} u(|L||\widehat{X}|)_{21}+O\left(u^{2}\right) .
\end{aligned}
$$

Together, (2.13) and (2.14) are equivalent to (2.11) with $j=1$, as required.

We can attempt a similar analysis for Method 2B. With the same notation as above, $X_{11}$ is computed by Method 2, so that

$$
\left|\widehat{X}_{11} L_{11}-I\right| \leq c_{r} u\left|\widehat{X}_{11}\right|\left|L_{11}\right|+O\left(u^{2}\right)=c_{r} u(|\widehat{X}||L|)_{11}+O\left(u^{2}\right),
$$

and $X_{21}$ is computed as $X_{21}=-X_{22} L_{21} X_{11}$. Thus

$$
\widehat{X}_{21}=-\widehat{X}_{22} L_{21} \widehat{X}_{11}+\Delta\left(\widehat{X}_{22}, L_{21}, \widehat{X}_{11}\right) \text {. }
$$

To bound the left residual we have to post-multiply by $L_{11}$ and use (2.15):

$$
\widehat{X}_{21} L_{11}+\widehat{X}_{22} L_{21}\left(I+\Delta\left(\widehat{X}_{11}, L_{11}\right)\right)=\Delta\left(\widehat{X}_{22}, L_{21}, \widehat{X}_{11}\right) L_{11}
$$

This leads to a bound of the form

$$
\left|\widehat{X}_{21} L_{11}+\widehat{X}_{22} L_{21}\right| \leq c_{n} u\left|\widehat{X}_{22}\right|\left|L_{21}\right|\left|\widehat{X}_{11}\right|\left|L_{11}\right|,
$$

which would be of the desired form in (2.5) if it were not for the factor $\left|\widehat{X}_{11}\right|\left|L_{11}\right|$. This analysis suggests that the left residual is not guaranteed to be small.

This difficulty with the analysis of Method 2B can be overcome by modifying the method so that instead of multiplying by $X_{j j}$ we perform a solve with $L_{j j}$. This gives the following variation:

\section{Method 2C.}

for $j=N:-1: 1$

$$
\begin{aligned}
& \left.\qquad X_{j j}=L_{j j}^{-1} \text { (by Method } 2\right) \\
& X_{j+1: N, j}=X_{j+1: N, j+1: N} L_{j+1: N, j} \\
& \text { Solve } X_{j+1: N, j} L_{j j}=-X_{j+1: N, j}
\end{aligned}
$$


For this method, the analogue of (2.16) is

$$
\widehat{X}_{21} L_{11}+\Delta\left(\widehat{X}_{21}, L_{11}\right)=-\widehat{X}_{22} L_{21}+\Delta\left(\widehat{X}_{22}, L_{21}\right),
$$

which yields

$$
\left|\widehat{X}_{21} L_{11}+\widehat{X}_{22} L_{21}\right| \leq c_{n} u\left(\left|\widehat{X}_{21}\right|\left|L_{11}\right|+\left|\widehat{X}_{22}\right|\left|L_{21}\right|\right)+O\left(u^{2}\right)
$$

Hence we have the following result.

Lemma 2.4 The computed inverse $\widehat{X}$ from Method $2 C$ satisfies

$$
|\widehat{X} L-I| \leq c_{n} u|\widehat{X}||L|+O\left(u^{2}\right)
$$

In summary, block versions of Methods 1 and 2 are available that have the same residual bounds as the point methods. However, in general, there is no guarantee that stability properties remain unchanged when we convert a point method to block form, as shown by Method 2B.

The analysis in this section can be modified to cater for the possibility that matrix multiplication and solution of a multiple right-hand side triangular system are done by "fast" techniques - for example, ones based on Strassen's method [12]. The appropriate changes to Lemmas 2.3 and 2.4 are to replace the absolute values by norms and to modify the constants. See [5] for details of this type of analysis.

Finally, in Table 2.1 we present some performance figures for inversion of a triangular matrix on a Cray 2. These clearly illustrate the possible gains in efficiency from using block methods, and also the advantage of Method 2 over Method 1. For comparison, the performance of a $k$ variant is also shown (both $k$ variants run at the same rate). The performance characteristics of the $i$ variants are similar to those of the $j$ variants, except that since they are row-oriented rather than column-oriented, they are liable to be slowed down by memory-bank conflicts, page-thrashing or cachemissing.

\section{$2.3 \quad$ Numerical Experiments}

In this section we describe some numerical experiments that provide further insight into the stability of the methods analysed above. The experiments were performed in 
Table 2.1: Mflop rates for inverting a lower triangular matrix on a Cray 2.

\begin{tabular}{ll|cccc} 
& & $n=128$ & $n=256$ & $n=512$ & $n=1024$ \\
\hline Unblocked: & Method 1 & 95 & 162 & 231 & 283 \\
& Method 2 & 114 & 211 & 289 & 330 \\
& $k$ variant & 114 & 157 & 178 & 191 \\
\hline Blocked: & Method 1B & 125 & 246 & 348 & 405 \\
(block size =64) & Method 2C & 129 & 269 & 378 & 428 \\
& $k$ variant & 148 & 263 & 344 & 383
\end{tabular}

MATLAB, which has a unit roundoff $u \approx 2.2 \times 10^{-16}$. We simulated single precision arithmetic of unit roundoff $u_{\mathrm{SP}}=2^{-23} \approx 1.2 \times 10^{-7}$ by rounding the result of every arithmetic operation to 23 significant bits. We regard the computed "double precision inverse" as being exact when computing forward errors.

One of the main aims of the experiments is to determine the behaviour of those left or right residuals for which we do not have bounds. If we find a numerical example where a residual is large then we are assured that it is not possible to obtain a small bound through rounding error analysis.

An important point to stress is that large residuals are hard to find! The examples we present were found after careful searching. We had to look at very ill-conditioned matrices to find interesting behaviour. Our experience ties in with the accepted fact that "The solutions of triangular systems are usually computed to high accuracy" $[17$, p. 150] — see [11] for an investigation of this phenomenon.

We present numerical results in Tables 2.2 and 2.3. For each method and matrix we tabulate left and right componentwise and normwise relative residuals, which in the "right" case are given by

$$
\min \{\epsilon:|L \widehat{X}-I| \leq \epsilon|L \| \widehat{X}|\} \quad \text { and } \quad \frac{\|L \widehat{X}-I\|_{\infty}}{\|L\|_{\infty}\|\widehat{X}\|_{\infty}}
$$

respectively. We also report the normwise relative error

$$
\frac{\left\|L^{-1}-\widehat{X}\right\|_{\infty}}{\left\|L^{-1}\right\|_{\infty}}
$$




\begin{tabular}{|c|c|c|}
\hline Method 1 & Comp'wise & Normwise \\
\hline left residual & $2.99 \mathrm{e}-4$ & $3.06 \mathrm{e}-5$ \\
\hline right residual & $8.35 \mathrm{e}-8$ & $2.23 \mathrm{e}-13$ \\
\hline relative error & $7.54 \mathrm{e}-2$ & $5.05 \mathrm{e}-4$ \\
\hline Method 2 & Comp'wise & Normwise \\
\hline left residual & $1.16 \mathrm{e}-7$ & $2.01 \mathrm{e}-9$ \\
\hline right residual & $5.61 \mathrm{e}-5$ & $7.50 \mathrm{e}-11$ \\
\hline relative error & $3.07 \mathrm{e}-2$ & $8.12 \mathrm{e}-4$ \\
\hline
\end{tabular}

and the componentwise relative error (for which we have no theoretical bounds)

$$
\min \left\{\epsilon:\left|L^{-1}-\widehat{X}\right| \leq \epsilon\left|L^{-1}\right|\right\}
$$

The reason for looking at the normwise quantities is that they may be small when the corresponding componentwise ones are large.

The matrix $L$ in Table 2.2 is the transpose of the upper triangular $Q R$ factor of the $15 \times 15$ Vandermonde matrix $V=\left(\alpha_{j}^{i-1}\right)$, where the $\alpha_{j}$ are equally spaced on $[0,1]$. We see that (2.1) is satisfied for Method 1 and (2.5) for Method 2, but not vice versa. It is interesting to note that both the normwise relative errors are three orders of magnitude smaller than the upper bound in (2.4).

For Table 2.3 we used a $10 \times 10$ matrix $L$ generated as the eighth power of a random lower triangular matrix with elements from the normal $(0,1)$ distribution. (This matrix is generated in MATLAB by the statements rand ('normal'), rand ('seed', 71), L = tril ( $\operatorname{rand}(10))$-8.) For each block method we used a fixed block size of 2. Table 2.3 confirms Lemmas 2.3 and 2.4. It also shows that both residuals can be large simultaneously for Method 2B; therefore the method must be regarded as unstable when the block size exceeds 1 . 
Table 2.3: $L=\operatorname{tril}(\operatorname{rand}(10))^{8}$.

$$
\kappa_{\infty}(L)=8.67 \mathrm{e} 12
$$$$
\operatorname{cond}(L)=3.41 \mathrm{e} 12, \operatorname{cond}\left(L^{-1}\right)=2.28 \mathrm{e} 11
$$

\begin{tabular}{l|ll} 
Method 1B & Comp'wise & Normwise \\
\hline left residual & $1.12 \mathrm{e}-2$ & $3.47 \mathrm{e}-3$ \\
right residual & $1.13 \mathrm{e}-7$ & $1.18 \mathrm{e}-9$ \\
relative error & $1.88 \mathrm{e}-1$ & $4.49 \mathrm{e}-2$
\end{tabular}

\begin{tabular}{l|ll} 
Method 2B & Comp'wise & Normwise \\
\hline left residual & $5.16 \mathrm{e}-2$ & $2.70 \mathrm{e}-3$ \\
right residual & $7.54 \mathrm{e}-2$ & $1.07 \mathrm{e}-3$ \\
relative error & $1.55 \mathrm{e} 1$ & $1.05 \mathrm{e} 0$
\end{tabular}

\begin{tabular}{l|ll} 
Method 2C & Comp'wise & Normwise \\
\hline left residual & $9.58 \mathrm{e}-8$ & $1.60 \mathrm{e}-8$ \\
right residual & $7.50 \mathrm{e}-2$ & $5.83 \mathrm{e}-4$ \\
relative error & $3.91 \mathrm{e}-1$ & $3.06 \mathrm{e}-2$
\end{tabular}




\section{Inverting a Full Matrix}

In this section we consider four methods for inverting a full matrix $A \in \mathbb{R}^{n \times n}$ given an $L U$ factorization computed by Gaussian elimination with partial pivoting (GEPP). We assume, without loss of generality, that there are no row interchanges. Recall that the computed $L U$ factors $L$ and $U$ satisfy (see, for example, [13])

$$
L U=A+E, \quad|E| \leq c_{n} u|L||U|
$$

\subsection{Method A}

Perhaps the most frequently described method for computing $X=A^{-1}$ is the following one.

\section{Method A.}

for $j=1: n$

Solve $A x_{j}=e_{j}$

end

Compared to the methods to be described below, Method A has the disadvantages of requiring more temporary storage and of not having a convenient block version. However, it is simple to analyse. Using (3.1) and (1.4) we find that

$$
\left(A+\Delta A_{j}\right) \hat{x}_{j}=e_{j}, \quad\left|\Delta A_{j}\right| \leq c_{n}^{\prime} u|L||U|+O\left(u^{2}\right),
$$

and so

$$
|A \widehat{X}-I| \leq c_{n}^{\prime} u|L||U||\widehat{X}|+O\left(u^{2}\right)
$$

This bound departs from the form (1.1) only in that $|A|$ is replaced by its upper bound $|L||U|+O(u)$. The forward error bound corresponding to (3.2) is

$$
\left|\widehat{X}-A^{-1}\right| \leq c_{n}^{\prime} u\left|A^{-1}\right||L||U||\widehat{X}|+O\left(u^{2}\right)
$$

\subsection{Method B}

Next, we consider the method used in LINPACK's routine xGEDI [6, Ch. 1]. 


\section{Method B.}

Compute $U^{-1}$ and then solve for $X$ the equation $X L=U^{-1}$.

To analyse this method we will assume that $U^{-1}$ is computed by an analogue of Method 2 or $2 \mathrm{C}$ for upper triangular matrices that obtains the columns of $U^{-1}$ in the order 1 to $n$. Then the computed inverse $X_{U} \approx U^{-1}$ will satisfy the residual bound

$$
\left|X_{U} U-I\right| \leq c_{n} u\left|X_{U}\right||U|+O\left(u^{2}\right)
$$

We also assume that the triangular solve from the right with $L$ is done by backward substitution. The computed $\widehat{X}$ therefore satisfies

$$
\widehat{X} L=X_{U}+\Delta(\widehat{X}, L)
$$

and so

$$
\widehat{X}(A+E)=\widehat{X} L U=X_{U} U+\Delta(\widehat{X}, L) U .
$$

This leads to the residual bound

$$
\begin{aligned}
|\widehat{X} A-I| & \leq c_{n} u\left(\left|U^{-1}\right||U|+2|\widehat{X}||L||U|\right)+O\left(u^{2}\right) \\
& \leq c_{n}^{\prime} u|\widehat{X}||L||U|+O\left(u^{2}\right)
\end{aligned}
$$

which is the left residual analogue of (3.2). From (3.4) we obtain the forward error bound

$$
\left|\widehat{X}-A^{-1}\right| \leq c_{n}^{\prime} u|\widehat{X}||L||U|\left|A^{-1}\right|+O\left(u^{2}\right) .
$$

Note that Methods A and B are equivalent, in the sense that Method A solves for $X$ the equation $L U X=I$ while Method B solves $X L U=I$. Thus the two methods carry out analogous operations but in different orders. It follows that the methods must satisfy analogous residual bounds, and so (3.4) can be deduced from (3.2).

We mention in passing that the LINPACK manual [6, p. 1.20] states that for Method B a bound holds of the form

$$
\|A \widehat{X}-I\| \leq d_{n} u\|A\|\|\widehat{X}\|
$$

This is incorrect, although counter-examples are rare (one is given in Table 3.2); it is the left residual that is bounded this way, as follows from (3.4). 


\subsection{Method C}

The next method that we consider appears to be new. It solves the equation $U X L=$ $I$, computing $X$ a partial row and column at a time. To derive the method partition

$$
X=\left[\begin{array}{ll}
x_{11} & x_{12}^{T} \\
x_{21} & X_{22}
\end{array}\right], \quad L=\left[\begin{array}{cc}
1 & 0 \\
l_{21} & L_{22}
\end{array}\right], \quad U=\left[\begin{array}{cc}
u_{11} & u_{12}^{T} \\
0 & U_{22}
\end{array}\right],
$$

where the $(1,1)$ blocks are scalars, and assume that the trailing submatrix $X_{22}$ is already known. Then the rest of $X$ is computed according to

$$
\begin{aligned}
x_{21} & =-X_{22} l_{21}, \\
x_{12}^{T} & =-u_{12}^{T} X_{22} / u_{11}, \\
x_{11} & =1 / u_{11}-x_{12}^{T} l_{21} .
\end{aligned}
$$

The method can also be derived by forming the product $X=U^{-1} \times L^{-1}$ using the representation of $L$ and $U$ as a product of elementary matrices (and diagonal matrices in the case of $U$ ). In detail the method is as follows.

\section{Method C.}

for $k=n:-1: 1$

$$
\begin{aligned}
& X(k+1: n, k)=-X(k+1: n, k+1: n) L(k+1: n, k) \\
& X(k, k+1: n)=-U(k, k+1: n) X(k+1: n, k+1: n) / u_{k k} \\
& x_{k k}=1 / u_{k k}-X(k, k+1: n) L(k+1: n, k)
\end{aligned}
$$

end

The method can be implemented so that $X$ overwrites $L$ and $U$, with the aid of a work vector of length $n$ (or a work array to hold a block row or column in the block case). Because most of the work is performed by matrix-vector (or matrix-matrix) multiplication Method $\mathrm{C}$ is likely to be the fastest of those considered in this section on many machines. (Some performance figures are given at the end of the section.)

A straightforward error analysis of Method $\mathrm{C}$ shows that the computed $\widehat{X}$ satisfies

$$
|U \widehat{X} L-I| \leq c_{n} u|U||\widehat{X}||L|+O\left(u^{2}\right)
$$

We will refer to $U \widehat{X} L-I$ as a "mixed residual". From (3.5) we can obtain bounds on the left and right residual that are weaker than those in (3.4) and (3.2) by a factor 
$\left|U^{-1}\right||U|$ on the left or $|L|\left|L^{-1}\right|$ on the right, respectively. We also obtain from (3.5) the forward error bound

$$
\left|\widehat{X}-A^{-1}\right| \leq c_{n} u\left|U^{-1}\right||U||\widehat{X}||L|\left|L^{-1}\right|+O\left(u^{2}\right),
$$

which is (3.3) with $\left|A^{-1}\right|$ replaced by its upper bound $\left|U^{-1}\right|\left|L^{-1}\right|+O(u)$ and the factors re-ordered.

The LINPACK routine XSIDI uses what is essentially a special case of Method C. This routine inverts a symmetric indefinite matrix $A \in \mathbb{R}^{n \times n}$ factored

$$
A=U D U^{T}, \quad U=U_{n} U_{n-1} \cdots U_{1},
$$

by forming the product

$$
A^{-1}=U_{n}^{-T} \cdots U_{1}^{-T} D^{-1} U_{1}^{-1} \cdots U_{n}^{-1}
$$

Here, $D=D^{T}$ is a block diagonal matrix with diagonal blocks of order 1 or 2 , and $U_{k}$ is a matrix differing from the identity above the diagonal in $s$ adjacent columns, where $s=1$ or 2 ; we have ignored the permutations required by the pivoting strategy. Analogously to (3.5) a residual bound holds of the form

$$
\left|U^{T} \widehat{X} U-X_{D}\right| \leq c_{n} u\left|U^{T}\right||\widehat{X}||U|+O\left(u^{2}\right),
$$

where $X_{D}$ is the computed inverse of $D$. Multiplying on the left by $D$, and using a bound for the left residual of $X_{D}$, we obtain

$$
\left|D U^{T} \widehat{X} U-I\right| \leq c_{n} u\left(|D|\left|U^{T}\right||\widehat{X}||U|+|D|\left|X_{D}\right|\right)+O\left(u^{2}\right) .
$$

\subsection{Method D}

The next method has been used in preliminary versions of the LAPACK routine xGETRI.

\section{Method D.}

Compute $L^{-1}$ and $U^{-1}$ and then form $A^{-1}=U^{-1} \times L^{-1}$.

The advantage of this method is that no extra workspace is needed; $U^{-1}$ and $L^{-1}$ can overwrite $U$ and $L$, and can then be overwritten by their product which is formed by steps analogous to those of $L U$ factorization. 
To analyse Method D we will assume initially that $L^{-1}$ is computed by Method 2 (or Method 2C) and, as for Method B above, that $U^{-1}$ is computed by an analogue of Method 2 or $2 \mathrm{C}$ for upper triangular matrices. We have

$$
\widehat{X}=X_{U} X_{L}+\Delta\left(X_{U}, X_{L}\right)
$$

Since $A=L U-E$,

$$
\begin{aligned}
\widehat{X} A & =X_{U} X_{L}(L U-E)+\Delta\left(X_{U}, X_{L}\right) A \\
& =X_{U} X_{L} L U-X_{U} X_{L} E+\Delta\left(X_{U}, X_{L}\right) A .
\end{aligned}
$$

Rewriting the first term of the right-hand side using $X_{L} L=I+\Delta\left(X_{L}, L\right)$, and similarly for $U$, we obtain

$$
\widehat{X} A-I=\Delta\left(X_{U}, U\right)+X_{U} \Delta\left(X_{L}, L\right) U-X_{U} X_{L} E+\Delta\left(X_{U}, X_{L}\right) A,
$$

and so

$$
\begin{aligned}
|\widehat{X} A-I| & \leq c_{n}^{\prime} u\left(\left|U^{-1}\right||U|+2\left|U^{-1}\right|\left|L^{-1}\right||L||U|+\left|U^{-1}\right|\left|L^{-1}\right||A|\right)+O\left(u^{2}\right) \\
& \leq c_{n}^{\prime \prime} u\left|U^{-1}\right|\left|L^{-1}\right||L||U|+O\left(u^{2}\right)
\end{aligned}
$$

This bound is weaker than (3.4) to the extent that $|\widehat{X}| \leq\left|U^{-1}\right|\left|L^{-1}\right|+O(u)$. Note, however, that the term $\Delta\left(X_{U}, X_{L}\right) A$ in the residual (3.8) is an unavoidable consequence of forming $X_{U} X_{L}$, and so the bound (3.9) is essentially the best possible.

The analysis above assumes that $X_{L}$ and $X_{U}$ both have small left residuals. If they both have small right residuals, as when they are computed using Method 1 , then it is easy to see that a bound analogous to (3.9) holds for the right residual $A \widehat{X}-I$. If $X_{L}$ has a small left residual and $X_{U}$ has a small right residual (or vice versa) then it does not seem possible to derive a bound of the form (3.9). However, we have

$$
\left|X_{L} L-I\right|=\left|L^{-1}\left(L X_{L}-I\right) L\right| \leq\left|L^{-1}\right|\left|L X_{L}-I\right||L|,
$$

and since $L$ is unit lower triangular with $\left|l_{i j}\right| \leq 1$, we have $\left|\left(L^{-1}\right)_{i j}\right| \leq 2^{n-1}$, which places a bound on how much the left and right residuals of $X_{L}$ can differ. Furthermore, since the matrices $L$ from GEPP tend to be well-conditioned $\left(\kappa_{\infty}(L) \ll n 2^{n-1}\right)$, and since our numerical experience is that large residuals tend to occur only for illconditioned matrices, we would expect the left and right residuals of $X_{L}$ almost 
always to be of similar size. We conclude that even in the "conflicting residuals" case Method D will, in practice, usually satisfy (3.9) or its right residual counterpart, according to whether $X_{U}$ has a small left or right residual respectively. Similar comments apply to Method B when $U^{-1}$ is computed by a method yielding a small right residual.

These considerations are particularly pertinent when we consider Method D specialized to symmetric positive definite matrices and the Cholesky factorization $A=$ $R^{T} R$. Now $A^{-1}$ is obtained by computing $X_{R}=R^{-1}$ and then forming $A^{-1}=X_{R} X_{R}^{T}$; this is the method used in the LINPACK routine xPODI [6, Ch. 3]. If $X_{R}$ has a small right residual then $X_{R}^{T}$ has a small left residual, so in this application we naturally encounter conflicting residuals. Fortunately, the symmetry and definiteness of the problem help us to obtain a satisfactory residual bound. The analysis parallels the derivation of (3.9), so it suffices to show how to treat the term $X_{R} X_{R}^{T} R^{T} R$ (cf. (3.7)), where $R$ now denotes the computed Cholesky factor. Assuming $R X_{R}=I+\Delta\left(R, X_{R}\right)$, and using (3.10) with $L$ replaced by $R$, we have

$$
\begin{aligned}
X_{R} X_{R}^{T} R^{T} R & =X_{R}\left(I+\Delta\left(R, X_{R}\right)^{T}\right) R \\
& =I+F+X_{R} \Delta\left(R, X_{R}\right)^{T} R, \quad|F| \leq\left|R^{-1}\right|\left|\Delta\left(R, X_{R}\right)\right||R|, \\
& =I+G,
\end{aligned}
$$

and

$$
|G| \leq c_{n} u\left(\left|R^{-1}\right||R|\left|R^{-1}\right||R|+\left|R^{-1}\right|\left|R^{-T}\right|\left|R^{T}\right||R|\right)+O\left(u^{2}\right) .
$$

From the inequality $\||B|\|_{2} \leq \sqrt{n}\|B\|_{2}$ for $B \in \mathbb{R}^{n \times n}$, together with $\|A\|_{2}=\|R\|_{2}^{2}+$ $O(u)$, it follows that

$$
\|G\|_{2} \leq 2 n^{2} c_{n} u\|A\|_{2}\left\|A^{-1}\right\|_{2}+O\left(u^{2}\right)
$$

and thus overall we have a bound of the form

$$
\|\widehat{X} A-I\|_{2} \leq d_{n} u\|A\|_{2}\|\widehat{X}\|_{2}+O\left(u^{2}\right) .
$$

Since $\widehat{X}$ and $A$ are symmetric the same bound holds for the right residual.

Returning to Method D for general matrices, we could obtain a forward error bound from (3.9), but a better one can be derived directly. We have, using (3.6) and $(2.6)$,

$$
\widehat{X}=\left(U^{-1}+\Delta_{U}\right)\left(L^{-1}+\Delta_{L}\right)+\Delta\left(U^{-1}, L^{-1}\right)+O\left(u^{2}\right)
$$


where

$$
\left|\Delta_{U}\right| \leq c_{n} u\left|U^{-1}\right||U|\left|U^{-1}\right|+O\left(u^{2}\right), \quad\left|\Delta_{L}\right| \leq c_{n} u\left|L^{-1}\right||L|\left|L^{-1}\right|+O\left(u^{2}\right) .
$$

Hence, using (3.1),

$$
\left|\widehat{X}-A^{-1}\right| \leq c_{n} u\left(\left|A^{-1}\right||L||U|\left|A^{-1}\right|+\left|U^{-1}\right|\left|L^{-1}\right||L|\left|L^{-1}\right|+\left|U^{-1}\right||U|\left|U^{-1}\right|\left|L^{-1}\right|+\left|U^{-1}\right|\left|L^{-1}\right|\right)+O\left(u^{2}\right) .
$$

This bound is broadly similar to (3.3).

\subsection{Numerical Results}

In terms of the above error bounds, there is little to choose between Methods A, B, $\mathrm{C}$ and D. We have run extensive numerical tests in MATLAB, evaluating the same residuals and forward errors as in section 2 (with $L$ replaced by $A$ in $(2.17)-(2.19)$ ). Thus, for example, the left componentwise and normwise residuals are given by

$$
\min \{\epsilon:|\widehat{X} A-I| \leq \epsilon|\widehat{X}||A|\} \quad \text { and } \quad \frac{\|\widehat{X} A-I\|_{\infty}}{\|\widehat{X}\|_{\infty}\|A\|_{\infty}}
$$

In Methods $\mathrm{B}$ and $\mathrm{D}$ we used Method 2 to compute $L^{-1}$ and $U^{-1}$. No significant difference of behaviour among the methods was observed. However, we make the following observations.

(1) The componentwise relative residuals can be large, as illustrated in Table 3.1, where

$$
A=\left[\begin{array}{cc}
I & B \\
B^{T} & 0
\end{array}\right], \quad A^{-1}=\left[\begin{array}{cc}
0 & B^{-T} \\
B^{-1} & -\left(B^{T} B\right)^{-1}
\end{array}\right],
$$

with $B$ a random $3 \times 3$ matrix with elements from the normal $(0,1)$ distribution. The bounds of this section do not guarantee small componentwise relative residuals. One reason is that $|L||U|$ may have nonzeros where $A$ has zeros, and so, for example, the right-hand side of (3.2) is not bounded by a multiple of $|A||\widehat{X}|$.

(2) Despite the observation in (1), we found that for all three methods both the left and right componentwise relative residuals are frequently at the unit roundoff level, and the normwise relative residuals are almost invariably at this level. An exceptional example is shown in Table 3.2. Here $A=L U$, where $U$ is 
the transpose of the matrix used in Table 2.3 and $L$ is the lower triangular factor from GEPP on a random matrix with elements from the normal $(0,1)$ distribution. In this example each method has a large normwise left or right residual.

Table 3.3 illustrates the effect of conflicting residuals. For the same matrix as in Table 3.2 we used Methods $\mathrm{B}$ and $\mathrm{D}$ with $L^{-1}$ and $U^{-1}$ computed by all possible combinations of Methods 1 and 2. The results confirm our prediction above that in practice it is the mode of computation of $U^{-1}$ that determines whether the left or right residual of the computed $A^{-1}$ is small.

Since all four methods have similar stability properties, the choice of method for LAPACK can be made on other grounds, namely performance and the amount of storage required. Method A has been ruled out because it does not allow the computed inverse to overwrite the $L U$ factors. Although Method D has the advantage of not requiring any extra working storage, its performance is significantly slower on some machines than Methods $\mathrm{B}$ or $\mathrm{C}$, because it uses a smaller average vector length for vector operations. In Table 3.4 we give some performance figures for a Cray 2, covering both blocked and unblocked forms of all three methods. A similar performance pattern is observed on an IBM $3090 \mathrm{VF}$, except that on that machine Method B is slightly faster than Method C. Although the blocked forms of Methods $\mathrm{B}$ and $\mathrm{C}$ require workspace to hold one block of columns, this is no more than many other block algorithms used in LAPACK, and is not considered a serious disadvantage. There is little to choose between Methods B and C; in the end Method B has been selected for the LAPACK routine xGETRI because it satisfies slightly cleaner error bounds, and because it has the virtue of tradition, being the method used in LINPACK. 
Table 3.1: $A=\operatorname{augment}(\operatorname{rand}(3))$.

\begin{tabular}{|c|c|c|}
\hline Method A & Comp'wise & Normwise \\
\hline left residual & $6.17 \mathrm{e}-1$ & $1.18 \mathrm{e}-8$ \\
\hline right residual & $8.06 \mathrm{e}-1$ & $1.42 \mathrm{e}-8$ \\
\hline relative error & $2.76 \mathrm{e} 8$ & $9.50 \mathrm{e}-8$ \\
\hline Method B & Comp'wise & Normwise \\
\hline left residual & $1.00 \mathrm{e} 0$ & $1.58 \mathrm{e}-8$ \\
\hline right residual & $1.00 \mathrm{e} 0$ & $2.26 \mathrm{e}-8$ \\
\hline relative error & $1.19 \mathrm{e} 8$ & $1.22 \mathrm{e}-7$ \\
\hline Method C & Comp'wise & Normwise \\
\hline left residual & $1.00 \mathrm{e} 0$ & $1.58 \mathrm{e}-8$ \\
\hline right residual & $8.06 \mathrm{e}-1$ & $2.23 \mathrm{e}-8$ \\
\hline relative error & $6.43 \mathrm{e} 7$ & $1.22 \mathrm{e}-7$ \\
\hline Method D & Comp'wise & Normwise \\
\hline left residual & $1.00 \mathrm{e} 0$ & $2.57 \mathrm{e}-8$ \\
\hline right residual & $1.00 \mathrm{e} 0$ & $2.13 \mathrm{e}-8$ \\
\hline relative error & $1.26 \mathrm{e} 8$ & $9.83 \mathrm{e}-8$ \\
\hline
\end{tabular}


Table 3.2: $A=L U$ with special $U$. $\kappa_{\infty}(A)=1.07 \mathrm{e} 9$ $\operatorname{cond}(A)=5.58 \mathrm{e} 8, \operatorname{cond}\left(A^{-1}\right)=4.24 \mathrm{e} 8$

\begin{tabular}{l|ll} 
Method A & Comp'wise & Normwise \\
\hline left residual & $9.37 \mathrm{e}-2$ & $7.88 \mathrm{e}-3$ \\
right residual & $1.55 \mathrm{e}-7$ & $8.08 \mathrm{e}-9$ \\
relative error & $4.64 \mathrm{e} 2$ & $1.59 \mathrm{e} 0$
\end{tabular}

\begin{tabular}{l|ll} 
Method B & Comp'wise & Normwise \\
\hline left residual & $8.62 \mathrm{e}-8$ & $2.26 \mathrm{e}-8$ \\
right residual & $6.57 \mathrm{e}-3$ & $1.13 \mathrm{e}-3$ \\
relative error & $4.64 \mathrm{e} 2$ & $1.59 \mathrm{e} 0$
\end{tabular}

\begin{tabular}{l|ll} 
Method C & Comp'wise & Normwise \\
\hline left residual & $1.83 \mathrm{e}-2$ & $2.24 \mathrm{e}-4$ \\
right residual & $2.08 \mathrm{e}-7$ & $1.29 \mathrm{e}-8$ \\
relative error & $4.64 \mathrm{e} 2$ & $1.59 \mathrm{e} 0$
\end{tabular}

\begin{tabular}{l|ll} 
Method D & Comp'wise & Normwise \\
\hline left residual & $1.41 \mathrm{e}-7$ & $4.15 \mathrm{e}-8$ \\
right residual & $6.57 \mathrm{e}-3$ & $1.13 \mathrm{e}-3$ \\
relative error & $4.64 \mathrm{e} 2$ & $1.59 \mathrm{e} 0$
\end{tabular}


Table 3.3: Normwise residuals.

\begin{tabular}{c|c|c|cc} 
& $\begin{array}{c}\text { Small left } \\
\text { residual }\end{array}$ & $\begin{array}{c}\text { Small right } \\
\text { residual }\end{array}$ & $\begin{array}{c}\text { Left } \\
\text { residual }\end{array}$ & $\begin{array}{c}\text { Right } \\
\text { residual }\end{array}$ \\
\hline Method B & $U^{-1}$ & & $2.26 \mathrm{e}-8$ & $1.13 \mathrm{e}-3$ \\
Method B & & $U^{-1}$ & $1.30 \mathrm{e}-5$ & $1.50 \mathrm{e}-8$ \\
Method D & $L^{-1}, U^{-1}$ & & $4.15 \mathrm{e}-8$ & $1.13 \mathrm{e}-3$ \\
Method D & $L^{-1}$ & $U^{-1}$ & $1.30 \mathrm{e}-5$ & $1.52 \mathrm{e}-8$ \\
Method D & $U^{-1}$ & $L^{-1}$ & $3.03 \mathrm{e}-8$ & $1.13 \mathrm{e}-3$ \\
Method D & & $L^{-1}, U^{-1}$ & $1.30 \mathrm{e}-5$ & $1.55 \mathrm{e}-8$
\end{tabular}

Table 3.4: Mflop rates for inverting a full matrix on a Cray 2.

\begin{tabular}{lc|cccc} 
& & $n=64$ & $n=128$ & $n=256$ & $n=512$ \\
\hline Unblocked: & Method B & 118 & 229 & 310 & 347 \\
& Method C & 125 & 235 & 314 & 351 \\
& Method D & 70 & 166 & 267 & 329 \\
\hline Blocked: & Method B & 142 & 259 & 353 & 406 \\
(block size =64) & Method C & 144 & 264 & 363 & 415 \\
& Method D & 70 & 178 & 306 & 390
\end{tabular}




\section{Conclusions}

Our conclusions are mainly positive ones. All but one of the methods considered here possess good enough error bounds that they are worthy contenders for practical use. The exception is the block method 2B for inverting a triangular matrix, which is unstable when the block size exceeds 1 .

Two general points arising from this work are worth emphasising, because they do not seem to be well known. First, for most of the inversion methods considered here only one of the left and right residuals is guaranteed to be small; which one depends on whether the method is derived by solving $A X=I$ or $X A=I$. Second, when a general matrix is inverted via an $L U$ factorization, the best form of residual bound holds only if the methods used for the " $L$-inversion" and the " $U$-inversion" satisfy residual bounds of the same parity - both methods must have a small left residual or both must have a small right residual.

Finally, we wish to stress that all the analysis here pertains to matrix inversion alone. It is usually the case that when a computed inverse is used as part of a larger computation the stability properties are less favourable, and this is one reason why matrix inversion is generally discouraged. For a simple example, let $L$ be the matrix of Table 2.2, $x=(1,1, \ldots, 1)^{T} / 3$, and $b:=L x$. We solved $L x=b$ in double precision in MATLAB by forward substitution and by forming $x=L^{-1} \times b$, where $L^{-1}$ was computed by Method 1 . For both computed solutions $\hat{x}$ we evaluated the normwise relative residual $\eta=\|L \hat{x}-b\|_{\infty} /\left(\|L\|_{\infty}\|\hat{x}\|_{\infty}+\|b\|_{\infty}\right)$. We found $\eta=7.15 \times 10^{-17}$ for forward substitution and $\eta=6.28 \times 10^{-12}$ for the inversion-based method, revealing a difference in stability of five orders of magnitude. 


\section{References}

[1] Y. Bard, Nonlinear Parameter Estimation, Academic Press, 1974.

[2] F.L. Bauer, Genauigkeitsfragen bei der Lösung linearer Gleichungssysteme, Z. Angew. Math. Mech., 46 (1966), pp. 409-421.

[3] C.H. Bischof, J.W. Demmel, J.J. Dongarra, J.J. Du Croz, A. Greenbaum, S.J. Hammarling and D.C. Sorensen, Provisional contents, LAPACK Working Note \#5, Report ANL-88-38, Mathematics and Computer Science Division, Argonne National Laboratory, Argonne, IL, 1988.

[4] R. Byers, Solving the algebraic Riccati equation with the matrix sign function, Linear Algebra and Appl., 85 (1987), pp. 267-279.

[5] J.W. Demmel and N.J. Higham, Stability of block algorithms with fast level 3 BLAS, LAPACK Working Note \#22 and Numerical Analysis Report No. 188, University of Manchester, England, 1990.

[6] J.J. Dongarra, J.R. Bunch, C.B. Moler and G.W. Stewart, LINPACK Users' Guide, Society for Industrial and Applied Mathematics, Philadelphia, 1979.

[7] J.J. Dongarra, F.G. Gustavson and A. Karp, Implementing linear algebra algorithms for dense matrices on a vector pipeline machine, SIAM Review, 26 (1984), pp. 91-112.

[8] G.E. Forsythe, M.A. Malcolm and C.B. Moler, Computer Methods for Mathematical Computations, Prentice-Hall, Englewood Cliffs, New Jersey, 1977.

[9] G.H. Golub and C.F. Van Loan, Matrix Computations, Second Edition, Johns Hopkins University Press, Baltimore, Maryland, 1989.

[10] N.J. Higham, Computing the polar decomposition-with applications, SIAM J. Sci. Stat. Comput., 7 (1986), pp. 1160-1174.

[11] N.J. Higham, The accuracy of solutions to triangular systems, SIAM J. Numer. Anal., 26 (1989), pp. 1252-1265. 
[12] N.J. Higham, Exploiting fast matrix multiplication within the level 3 BLAS, ACM Trans. Math. Soft., 16 (1990), pp. 352-368.

[13] N.J. Higham, How accurate is Gaussian elimination?, in Numerical Analysis 1989, Proceedings of the 13th Dundee Conference, Pitman Research Notes in Mathematics 228, D.F. Griffiths and G.A. Watson, eds., Longman Scientific and Technical, 1990, pp. 137-154.

[14] P. McCullagh and J.A. Nelder, Generalized Linear Models, Second Edition, Chapman and Hall, 1989.

[15] W. Miller and D. Spooner, Software for roundoff analysis, II, ACM Trans. Math. Soft., 4 (1978), pp. 369-387.

[16] R.D. Skeel, Scaling for numerical stability in Gaussian elimination, J. Assoc. Comput. Mach., 26 (1979), pp. 494-526.

[17] G.W. Stewart, Introduction to Matrix Computations, Academic Press, New York, 1973.

[18] J.H. Wilkinson, Error analysis of direct methods of matrix inversion, J. Assoc. Comput. Mach., 8 (1961), pp. 281-330.

[19] J.H. Wilkinson, Rounding Errors in Algebraic Processes, Notes on Applied Science No. 32, Her Majesty's Stationery Office, London, 1963.

[20] J.H. Wilkinson, The Algebraic Eigenvalue Problem, Oxford University Press, 1965 . 